\title{
Building a Business on Open Source Software
}

\author{
Anthony I. Wasserman \\ Carnegie Mellon Silicon Valley \\ Mountain View, CA 94035 USA \\ Email: tonyw@sv.cmu.edu
}

\begin{abstract}
For many years, free and open source software was exclusively associated with the research community, in contrast to the closed source products developed by commercial software vendors. Over the past decade, there has been a vast increase in the availability of open source software, growing from such projects as Linux and Apache. Open source software is being widely adopted in industry, which has led to numerous business opportunities for entrepreneurs and vendors. This activity is having a disruptive effect on the software industry that has affected even the largest software vendors. This paper describes the emergence of open source business, the growing commercialization of open source, and some business models that can be used to build a successful business based on open source software.
\end{abstract}

\section{Introduction}

The notions of free and open source software go back to the earliest days of computing, when all software was free and source code was routinely published. In the late 1960s, though, IBM unbundled software from hardware, charging money for the operating system and other software. At about the same time, new software companies arose, building businesses around the licensing and support of software systems, such as database management systems. Software developed in the research community continued to be freely distributed at no charge, even as commercial software grew to a multi-billion dollar industry. Much of the foundation for the Internet was funded by the US Government, with all of the source code freely available.

In 1985, Richard Stallman at MIT put forth the notion of free software based on his personal belief that software should be free. He created the Free Software Foundation (FSF) not just as a technical movement but also as a social, political, and economic movement. In the early 1990s, Linus Torvalds, a student in Finland, first developed the Linux operating system, and made it freely available under the GNU General Public License developed by Stallman and the FSF. Linux became extremely popular, especially among hobbyists, who created a large and active community that enhanced Linux, eventually making it sufficiently reliable for widespread commercial use.

Before companies would use Linux or any other free software, they wanted to 
make sure that there were commercial sources of training and support. In the late 1980s, Cygnus Solutions was created to provide such support. Cygnus was able to build a profitable business not by selling software, but by selling services to help people use free software. Cygnus was eventually acquired by Red Hat, which has become the leading commercial vendor of Linux software. There was a growing interest in commercialization of open source software which led to the 1998 Open Source Definition (Perens, 1998). This definition included a set of approved licenses which are managed by a formal organization, the Open Source Initiative (OSI). The OSI is responsible for reviewing and approving open source licenses, as well as promoting open source development and use.

The notion of open source software has three important components. The first is that all of the code and all of the scripts and libraries needed to run the program are made available, without restriction, to anyone who wants access to that code. The second is that the software is licensed under a mechanism that defines appropriate uses for the program and the rules for distributing changes to the source code. Finally, the software is supported by a community of interest, including contributors to the source code. There is little value to software that is released without a community of people to maintain it and test it.

The growth of open source software has transformed the software industry. Many companies now release the source code of the software and make it available under an approved open source license, rather than selling traditional proprietary (closed source) licenses.

\section{The State of Open Source Software}

Today, there is a vast range of open source software available, not just for Linux, but also for Windows and MacOS X. The software includes not only infrastructure software and development tools, but also applications software. At the infrastructure level, it is easy to find highly reliable open source software as Web servers, application servers, system management tools, and virtualization tools. Among development tools, there are two major integrated development environments, Eclipse and NetBeans, both of which can be used for a wide variety of software development tasks. In addition, both of those development environments are frameworks that support the addition of tools, not necessarily open source, developed by others.

At the application layer, there are not only widely used tools for word processing, e.g., OpenOffice, e-mail, e.g., Zimbra and Thunderbird, and web browsing, e.g., Firefox, but also more specialized applications for accounting, image editing, and telephony. More and more of these applications are being developed and supported by new companies, which also provide support, training and the promise of ongoing maintenance. That is a strong contrast with traditional community-based development where there was no such assurance.

There are at least four different kinds of open source projects. The first is a community-based project, started by a small group and developed independently of 
any organization or company. The developers on these projects often work as volunteers in their free time. The second is a community-based project under the structure of a non-profit organization, such as the Apache Foundation. The Foundation leadership oversees progress of the project and provides some support. Project developers may work as volunteers, or may be paid by a company to contribute. The third is a project with both community and commercial aspects. In this case, the project may be led by a company, and many of the contributors may be paid by that company to work on the project. However, participation in the project and the group of committers is open to all. The last is a project started by a company that is creating a commercial business around the open source software. In this case, the key decisions about the project are made by company management, and all of the developers work for the company, with little opportunity for community volunteers to contribute code.

From a business perspective, there are significant differences among these models. The projects with significant commercial participation often have a more clearly defined project roadmap, a systematic testing process, and mechanisms for both commercial and non-commercial support.

The remainder of this paper focuses on a variety of business models by which people can build successful businesses on open source software.

\section{Toward Commercial Opportunities}

The title of this paper includes two major options: creating a business that offers services related to open source software, and creating a business that uses open source software to deliver an application or system. In both cases, an important prerequisite is that the open source software be of high enough quality that companies will adopt it for their routine and critical business operations. Now that there are many software products that meet that criterion, a large number of possibilities for commercialization arise.

These opportunities are driven, in part, by user unhappiness with traditional software and fee structures. Commercial licenses require the payment of substantial initial fees followed by ongoing support costs. By contrast, open source software has no initial fee and allows an organization to evaluate that software until such time as it is ready to be deployed for commercial purposes. At that time, and only at that time, must the user enter into a commercial agreement with the vendor and pay fees. For most companies, and especially for startups, the savings on the initial license fee is extremely significant.

In addition, companies are looking to save money in general and are beginning to consider open source alternatives as they evaluate different categories of software. Perhaps surprisingly, some users of open source software, especially companies, want to pay and expect to pay for use of that software, as a way to assure that they can obtain service as needed.

These factors, taken together, are transforming the software industry for both vendors and buyers. As one example, established vendors of proprietary closed 
source products increasingly find themselves competing against open source alternatives. These vendors have responded in several different ways: 1) by offering basic versions of their product at no charge, and hoping to sell support services to those users; 2) by using the offer of the free basic version as a way to build a relationship with users, who may eventually license the paid commercial version, and; 3) acquiring commercial open source companies that enable them to offer an open source alternative and support services to those users who don't want to pay the initial license fees for the proprietary software product.

As another example of industry transformation, software-intensive startups are making extensive use of open source software as building blocks for their products and services. That approach sharply reduces the time, effort and cost needed to bring their product to market, thereby reducing the amount of needed outside investment.

Finally, companies have chosen to release their software under an open source license rather than keeping it closed and distributing it under a proprietary license. For established companies, there are good reasons for choosing to develop and release code under an open source license; Goldman and Gabriel (Goldman and Gabriel, 2005) describe the experience of doing so at Sun Microsystems.

For new companies, the open source strategy allows them to build a user base quickly, beat their competitors on price, and save significantly on sales and marketing expenses. Over time, they develop ways to generate revenue from their software, using one or more of the approaches described in Section 4. Unlike communitybased open source projects, such as the Apache http server, these commercial open source startups control the development process and effectively own the source code.

In short, the emergence of open source software has affected almost every aspect of the software business, forcing established software vendors to respond to the competitive pressures of open source software, and creating new types of business opportunities that build on open source software.

\section{Business models using open source software}

There are many ways by which an individual, a partnership, or a company can create a business related to open source software. In some cases, it is not necessary for the business to have any particular role in development and maintenance of the code. Weber (Weber, 2004) has contrasted businesses based on proprietary software with those based on open source software, listed some "basic business model templates", and described the ways that six different companies have used one or more of these ideas in their businesses.

Fink (Fink, 2003) described seven different business models in his survey work on open source software, including selling proprietary software on the open source Linux platform and "open sourcing" legacy proprietary software; these ideas have not been successful and are not included here. Krishnamurthy (Krishnamurthy, 2005) identified six different business models that built on open source software, with particular emphasis on selling support services. As we shall see, the marketplace has evolved to present additional options. 
In this section, we describe many of the most effective ways to create a successful business using open source software, giving specific examples where possible.

\subsection{Subscription Model}

The most widely used business model for open source software is a subscription model, where the user pays a fee for regular updates to the software. In this model, there is usually no requirement that a user of the software purchase a subscription, so a user can download software and use it indefinitely at no charge. (RedHat Enterprise Linux is a notable exception to this statement.) The user can manually check for software updates or new releases, install them, and use discussion forums for answers to technical problems, again at no charge. In addition, a user can hire consultants and contractors to help with specific issues. Excellent examples of this model are provided by Novell with their SuSE Linux Enterprise Desktop and by RedHat with the JBoss application server.

From a business perspective the subscription model offers some valuable advantages. For a supplier, this current model provides a more predictable revenue flow than is possible with a traditional license model. For customers, it is only necessary to pay for the subscription when they can generate revenue based on the use of that software.

\subsection{Offering Commercial and Open Source Products}

Another approach for a vendor is to offer both open source and commercial products. The commercial products can be licensed in the traditional way, or as an "on demand" service, and are typically closed source. They can provide significant revenue for the business, which may also offer open source products under other business models or even free of charge.

Among the vendors following this approach are CollabNet, with its Enterprise Edition and the open source Subversion configuration management system. Similarly, JasperSoft offers a commercial JasperServer Business Intelligence suite, as well as an open source community edition of the JasperServer reporting tool.

Krugle offers a non-commercial search mechanism for open source software through their krugle.org site. That site was developed to generate awareness for their commercial enterprise product, aimed at helping companies manage their internal software assets.

\subsection{Support and Training Model}

In addition to selling subscriptions, a common business model involves providing support and training for open source products. Training may come in the form of textbooks and instructional material, such as offering of courses via live training or video.

Textbooks can be a profitable model, but it is important to note that there is a large upfront cost in writing the manuscript, publishing it, and making it available for 
distribution. Nonetheless, there are several publishers, particularly O'Reilly and aPress, who have created a substantial library of books covering various open source software projects.

Some people and organizations have also created small businesses by distributing media, i.e., DVDs and CDs, with various open source components. This service is valuable in places where connection speeds are slow and where the charges for transferred data is high. However, this approach does not seem to generate enough revenue or profit to be a sustainable business model.

Training courses can be profitable and can be prepared in relatively little time, especially compared to the time needed to write and publish a book. However, it often takes considerable effort to market the courses to an audience that is large enough to help create a profitable business.

Technical support can be offered as fee-based help or as an ongoing subscription for customers who need someone to call for help with problems and issues that they may have with open source software. Many companies have an IT policy that they will not use any software (open source or not) for which they cannot obtain a support contract with service level agreements appropriate for the criticality of that software. In many cases, an open source product may obtain more support contracts when it is acquired by a larger company with extensive geographical coverage, as happened after Oracle's acquisition of Sleepycat Software (BerkeleyDB) and Sun's acquisition of MySQL AB.

In all of these cases, it is possible to create a business around one or more software projects without having contributed to the development of that software. This approach is particularly useful when customers need local support, without having to wait for an email reply from a distant organization on a different time zone. Also, since English is so widely used for software and its documentation, it is sometimes difficult to obtain high-quality support in local languages. As software is increasingly localized for these languages, there is a growing need for support in those local languages, making it possible to create a successful business around support and training.

\subsection{Dual License Model}

Another business alternative is to offer open source software under a dual license model, i.e., allowing the customer to select the license that will apply for its use of the software. In this approach, users can download and freely use the open source software for non-commercial purposes, including evaluation for commercial suitability and development of their products and/or services. When they are ready to offer that software in a product that is licensed or sold to customers, they can then switch licenses, obtaining a commercial license to replace the non-commercial one.

MySQL AB is one company that took this dual license approach. Before they increased their commercial marketing efforts in their last years as an independent company, there were as many as 1000 non-commercial users of MySQL for every commercial one. The revenue from the commercial licenses grew quickly in the past few years and supported the growth of the company which Sun Microsystems 
acquired in 2008 for $\$ 1$ billion US.

In some cases, the software offered in the commercial version offers additional options or features not available in the "community", i.e., open source, version. Users can start out with the open source version, but often find that they need the additional features when they want to use the software in a production environment.

\subsection{Hosted Service}

In a hosted service model, a vendor can use open source software to create software that can be offered as a service. There are numerous examples of such a hosted service including web searching, document editing, project management, and content management systems. For a software startup, using open source software to build a hosted service is extremely helpful. First, there is no initial cost for software licenses, but more importantly, the startup can focus on the added value that it brings to its service without having to redevelop database management systems or other infrastructure components that will be used to implement their service.

Some of the largest and best-known companies have begun by using open source software. Yahoo, for example, built their directory service and other websites on top of a BSD Unix-based open source operating system. Google, as another example, has made extensive use of open source software, particularly the Apache http server, which they have greatly modified to meet their specific needs. Many non-commercial sites also used a combination of open source software to provide their service.

Many of the open source wikis and content management systems employ several different open source components to provide their service, which others then use as the basis for their sites. The Mozilla Foundation created the SpreadFirefox website as a marketing tool for the open source Firefox browser. The website is built using the open source Drupal content management system, which in turn uses the Apache http server, the MySQL database, and the PHP scripting language. That site, in turn, serves as a directory for numerous other Firefox-related sites, virtually all of which are built with similar open source tools.

Beyond those examples, open source software is frequently used to create software-as-a-service (SaaS) offerings for which customers may purchase subscriptions. Among the many instances of such SaaS products are the BasecampHQ project management system and the SugarCRM customer relationship management system. BasecampHQ is built with Ruby on Rails, while SugarCRM uses the Apache http server, a relational database management system, and PHP. Customers don't pay for the source code, but rather for access to the SaaS application. SugarCRM is particularly interesting, since there is a community open source version, a professional version (where some of the code is not open), and an SaaS version, with the latter two producing revenue for the company.

\subsection{Advertising Model}

While many of the examples cited above present a direct revenue opportunity, e.g. from subscriptions or paid services, it is also possible to generate revenue from 
advertising, as has been amply demonstrated in the market. For example, targeted ads can be displayed on the site where the user accesses an SaaS application. A financial services site could connect to an advertising network, such as Google's AdWords, and deliver ads that are relevant to someone looking for investment opportunities or professional assistance with finance. It is easy to imagine analogous types of ads for open source SaaS applications in other application domains. Of course, this advertising model is already widely used for web applications built on proprietary software, but applies just as well for web applications built on open source software.

An open source-based SaaS application could offer both a paid subscription version and a free version, with advertising displayed on the free version. In this way, the developer of the application is able to monetize all users of the application, using two different approaches, subscriptions and advertising, to do so.

A related approach is a "sponsored" site, where a company pays for the ongoing cost of an SaaS application built on open source software. Thus, a financial services company might sponsor a site related to retirement planning, or an airline might sponsor a travel-related site that might bring them additional passengers.

\subsection{Packaging Model}

There are many thousands of open source packages available, many of which, e.g., libraries, are only useful in combination with other software. While developers are content to work with individual packages, users are more interested in solutions that may involve combining several different components into a useful grouping. One such example, noted above, is Drupal, which combines a Web server, a database management system, and the PHP scripting language. The Drupal community has created various modules for such activities as blogging, discussion forums, and social networking, along with themes to customize site appearance. Taken together, these pieces provide a simple way to quickly deploy a new site with little or no software development.

Many such packages are becoming available, often with no initial cost. BitRock has sponsored the BitNami site (http://www.bitnami.org), which has created installation packages for more than twenty different applications, including seven content management systems, three issue tracking systems, two wikis, and more. A user can download one of these packages, and the installer will automatically install the various open source components needed to use the chosen application. The availability of such packages greatly simplifies the user's task. Not only is it unnecessary to locate the individual components needed for the application, but the components have been configured to work together for that application.

Along the same lines, OpenLogic has created OpenLogic Exchange, aimed at helping enterprises find and download open source packages. OpenLogic offers support services to generate revenue from this service. SpikeSource also offers integrated and certified open source packages as part of their Solutions Factory, along with a subscription-based SpikeIgnite program that offers pre-packaged stacks. 


\subsection{Commercial Enhancement}

Many open source software projects were not developed with commercial requirements in mind. However, depending on the license associated with the project, it is possible to extend those projects and deliver a commercial product based on enhancements to the underlying software. While most commercial enhancers of open source software contribute the software changes back to the original project, some licenses, such as BSD, do not require that this be done. In that situation, the company that makes the commercial enhancement is free to license it on either open source or commercial terms using any business model that seems appropriate.

For example, there are several companies that have enhanced the Postgres database management system. One such project called PowerGres, from SRA, adds high-availability and scalability features to Postgres. Another such product, called Postgres Plus, from EnterpriseDB, offers binary downloads (not source code) of their enhanced version of Postgres. That product is offered at no charge for nonproduction use, with a commercial license available for production use. Taking an open source program proprietary, i.e., "closing" it, is often seen as going against the spirit of open source software, even if doing so is permitted by the associated license.

\subsection{Embedding Model}

Open source software can also be used as part of a hardware/software product, with the software embedded in the hardware. Because of the licensing requirements on open source software, the product manufacturer must make the [potentially modified] open source software available to anyone who is interested. The manufacturer creates a business based on selling the manufactured product with no intent to generate revenue from the open source software. In this case, the manufacturer is simply taking advantage of the open source software to speed development of the embedded product.

There are numerous examples of products built on open source software. One is the TiVo digital video recorder. The Software Development Kit for the TiVo Home Media Engine is available as a project on the SourceForge repository. Another is Amazon.com's Kindle reader device, built on Linux, with the source code for the relevant packages available on Amazon.com's website. Another example is the OpenMoko FreeRunner mobile telephone, in which the NeoSoftwareStack is embedded. That stack includes various open source components, including graphical toolkits, a Java Virtual Machine, the GNU C compiler, and many others, all built on a Linux operating system. Finally, most of the routers developed by the Linksys subsidiary of Cisco Systems are based on open source software. The technical support area of the Linksys web site contains a list of those routers with a link to the source code for each router, in keeping with the requirements of the GPL license, under which the code was licensed.

In addition to the multitude of traditional computers on which an open source operating system, e.g., Linux or FreeBSD, can be installed, there is also a growing category of devices on which Linux is preinstalled and serves as the de facto operating environment for the device. One example is the Nokia N800/N810 Internet tablet 
family, for which the Maemo operating system (a Linux variant) serves as the platform. Another, better known, device is the XO Laptop, created by the One Laptop per Child Foundation, which is based on the open source Fedora distribution of Linux, sponsored by RedHat. In both cases, the base operating system is augmented by hundreds of additional open source software components, producing a complete operating environment with its own unique characteristics. Different versions of Linux serve as the embedded operating system in many of the new, lightweight "netbooks", such as the Asus EEE, the MSI Wind, and the HP MiniNote, as well as in mobile telephones, including those compliant with the specifications of the LiMo Foundation. .

Many open source software components have been developed specifically for use in embedded systems, including versions of Linux optimized for real-time and embedded systems.

\subsection{Consulting and Integration Strategy}

With a consulting strategy, a vendor does not offer open source software directly but instead helps clients to make strategic decisions and investments related to open source. Consultants charge for their time and services, which may also include installation, integration, and customization of software, both open source and proprietary.

Consulting services come in all sizes, ranging from tiny one and two-person operations to the multi-billion dollar IBM Global Services. Some open source developers can make their living by offering consulting services on the open source projects that they have developed. Many of the larger firms, known as system integrators, put together solutions for their business clients. Historically, these solutions have been built almost exclusively on proprietary commercial software; now, however, open source software has become an important component of these solutions. Riehle (Riehle, 2007) describes the system integrator perspective and how their use of open source software improves their business.

For example, a consultant may help with the evaluation of a database management system or a content management system. The selection of the database management system may be followed by application design and schema design, which can be very beneficial to the consulting firm. Other consulting firms, known as industry analysts, don't do any software development at all. Instead, they produce independent reports on technological trends, and may evaluate products in specific categories and/or organize conferences to report and discuss their findings. For many of these analyst firms, open source software is just one of many lines of practice. Clients of the analyst firms may also receive individual advice, which helps them to make business decisions.

Consulting is a particularly effective business model for individual entrepreneurs, who can work with local companies to help them make the most effective use of open source software. This type of consulting is particularly valuable for small and medium-sized businesses, who lack the resources to use expensive proprietary software or to pay high-priced consulting firms. 
In addition to individual product consulting, there is also a need for strategic consulting related to open source software. Many large corporations need advice on how to evaluate adopt and use open source software, including modification of their IT policies, education about open source licenses, and creation of open source contribution policies for their employees. These companies have many years of experience with evaluation and acquisition of proprietary software, but open source is new to them.

\subsection{Patronage Model}

The patronage model involves contribution of people, equipment, time, and/or money to open source projects, typically by large corporations. These corporations do not directly receive any revenue from their participation in these projects. However, there are several very good reasons for these companies to contribute resources to open source projects.

First, these companies are often looked upon more favorably because of their contributions. Second, by giving away some of their software, they increase visibility of their own company in the open source community, which may help them to sell their other products and services, including those unrelated to the open source software. Third, as they pay their employees to work on open source projects, their employees become aware of new tools and techniques that may increase their value to the company. Furthermore, their employees work closely with other contributors to open source projects, which helps them to identify people who might make good employees. Thus, the patronage role also serves as a useful recruiting mechanism.

In many cases, the extent of the contribution is very substantial. For example, IBM is the major supporter of the Eclipse development environment. Similarly, Sun Microsystems is a leading supporter of OpenOffice, NetBeans, and GlassFish. Many companies contribute to the Mozilla Foundation in support of the Firefox browser and Thunderbird e-mail product.

In addition to the paid employees, many individuals also volunteer their time to these large and established products. There are many reasons for doing so, including the sense of belonging to a community, an alternative outlet for creative activities aside from their other activities (possibly including a programming job), and a way to build their own professional credentials, perhaps as an entry on their resumé or a personal contact that could help them to obtain new employment, contracting, or consulting work. For an individual, giving time and effort to a project can lead to business opportunities.

Finally, it should be noted that not all of this patronage is entirely benevolent. If a company supports the enhancement of an open source operating system or suite of development tools, their intent may include taking business away from commercial vendors of proprietary operating systems and development tools. Even so, the net result is usually beneficial to those who are interested in having high quality open source software. 


\section{Starting a new open source business}

Given this wide range of business models, one could naturally question which of these models present good opportunities for new businesses. There is, of course, no answer that fits everyone. Instead, there are many factors that influence the choice, including not only one's technical and managerial skills and interests, but also available funding and geographical location, which influences market opportunities.

For someone with some technical background, combined with an interest in working with users, it is often easiest to create a business around delivering services on one or more open source projects, particularly in locations outside of North America and Western Europe. Startup costs are low, competition is limited, and the service can provide high value to governments, as well as to small and medium sized businesses. As an adjunct to creating the training and consulting service, it's essential to become a member of the communities for the various open source projects, though not necessarily a source code contributor.

Similarly, it is relatively easy to create a business that applies packaged open source software to the development of web sites for small and medium sized businesses. It's quite straightforward to use the Drupal or Joomla content management systems to create an e-commerce website or a community message center. Both of these small businesses allow the business principal(s) to get started quickly at low cost, and to offer services to customers very quickly.

On the other hand, neither of these small business ideas will lead to a large business opportunity, given their labor-intensive nature. For those opportunities, one must create something unique that can be used by a larger number of people. Both software products and embedded systems fall into this category.

In the realm of software products, the infrastructure and development tools categories are more fully addressed than are applications. One particularly interesting area is games, which can be built using an SaaS model. The area of "casual gaming" is growing extremely fast, and the most popular games earn large sums of money for their creators and publishers, either through a subscription model or through an ad-supported site. In addition, there are many opportunities involving the development of SaaS applications built on an open source infrastructure.

Mobile applications are another important category, less fully developed than some of the others mentioned here. All of the major mobile handset vendors are including Linux-based devices in their product lines, creating an opportunity for downloadable applications, including games, that will run on those devices. The business possibilities seem most promising for those applications that take advantage of the unique properties of mobile devices, including location-based services, motion-sensing, and mobile social networking based on proximity.

Of course, the above suggestions are just a starting point, and some of them may turn out not to be so attractive over time, particularly as more people build open source applications and commercialize them. As with any other business concept, entrepreneurs must consider the market opportunity, including size and competition, as key steps in their initial analysis. 
In conclusion, this collection of ideas for building a business on open source software ranges from those that can be undertaken by a single individual to those that may need a substantial organization with a significant financial investment. There are, undoubtedly, many other concepts that can and will be explored.

\section{References}

Fink, M. (2003), The Business and Economics of Linux and Open Source. Englewood Cliffs, NJ: Prentice-Hall.

Goldman, R. and R. Gabriel (2005), Innovation Happens Elsewhere. San Francisco: Morgan Kauffman.

Krishnamurthy, S. (2005), 'An Analysis of Open Source Business Models', in J. Feller et al. (eds.), Perspectives on Free and Open Source Software. Cambridge, MA: The MIT Press, pp. 279-296.

Perens, B. (1998), 'The Open Source Definition', reprinted in C. DiBona, S. Ockman and M. Stone (eds.) (1999), Open Sources: Voices from the Open Source Revolution. Sebastopol, CA: O'Reilly. (Available online at http://www.oreilly.com/catalog/opensources/book/perens.html, accessed May, 2008)

Riehle, D. (2007), 'The Economic Motivation of Open Source Software: Stakeholder Perspectives,' IEEE Computer, 40 (4), 25-32. (Available online at http://www.riehle.org/computer-science/research/2007/ computer-2007-article.html, accessed May, 2008)

Weber, S. (2004), The Success of Open Source. Cambridge, MA: Harvard University Press.

\section{Acknowledgments}

The author gratefully acknowledges the valuable comments of Bob Bickel and Dirk Riehle on an earlier version of this paper. 